http://jmscr.igmpublication.org/home/ ISSN (e)-2347-176x ISSN (p) 2455-0450 crossref DOI: https://dx.doi.org/10.18535/jmscr/v7i9.54

\author{
Dournal Of Medical Science And Clinical Research \\ IGM Publication \\ An Official Publication of IGM Publication
}

\title{
Spina Ventosa: Tuberculous Dactylitis in a 8-year-old boy- A Rare Case Report
}

\author{
Authors \\ Dr Sunil Kumar Agarwalla ${ }^{1}$, Dr Anjali Saji ${ }^{2 *}$ \\ ${ }^{1}$ Associate Professor, Dept of Pediatrics, M.K.C.G Medical College, Berhampur, Ganjam, Odisha, 760004 \\ ${ }^{2}$ Junior Resident, Dept of Pediatrics, M.K.C.G Medical College, Berhampur, Ganjam, Odisha, 760004 \\ *Corresponding Author \\ Dr Anjali Saji \\ Address: PG Ladies Hostel, M.K.C.G Medical College, Berhampur, Odisha, 760004, India
}

\begin{abstract}
Tuberculous infection of metacarpals, metatarsals, and phalanges of hands and feet is known as tuberculous dactylitis. It forms a spindle shaped expansion of short tubular bones due to tuberculous granuloma. TB dactylitis and spina ventosa are the common variants of TB osteomyelitis seen in children below five years. In our case an 8-year-old boy was admitted with swelling and flexion deformity of metacarpals and fingers of right hand and discharging sinuses. The child was investigated in line of tuberculosis and diagnosed to have tuberculous dactylitis. The patient responded well to anti-tubercular therapy.

Keywords: Tuberculous Dactylitis, Tuberculous Granuloma, Spina Ventosa.
\end{abstract}

\section{Introduction}

Commonly TB infection of bone presents as TB spine (Pott's spine). Tuberculous infection of short tubular bones of hands and feet is known as tuberculous dactylitis. ${ }^{(1)}$ It is estimated, rarely, i.e. $1-6 \%$ of children with primary infection may develop bone and joint tuberculosis in 1-3 years. Nearly $85 \%$ of these patients are below 6 years. ${ }^{(2)}$ The hand is more frequently involved than the foot. Proximal phalanx of index and middle fingers are the most common site of involvement. It often follows a mild course without fever or signs of inflammation unlike osteomyelitis. The term 'spina ventosa' derives from 'spina' meaning 'short bone' and 'ventosa' meaning 'expanded with air' because of the cystic expansion of the short tubular bones. ${ }^{(3)}$ There may be a gap of few months to years from time of initial infection to the point of diagnosis. The diagnosis is delayed because osseous tuberculosis is a paucibacillary lesion. ${ }^{(4)}$

\section{Case Report}

\section{Clinical Features}

A 8 year old boy was presented initially with swelling of dorsum of right hand and swelling of right thumb and ring finger for the last 4 months. The swelling was initially small and gradually increased in size and later developed discharging sinuses. Initially he consulted a local hospital after a month of his presenting complaints and given a course of oral antibiotics. But the swelling did not subside with those medication. The swelling not only persisted but also caused restricted 
movements of fingers. There was no history of fever, night sweats, weight loss or trauma or contact with any tuberculosis.

Local examination revealed two fusiform swelling of size about $4 \mathrm{~cm} * 3 \mathrm{~cm}$ on the dorsum of thumb involving proximal interphalangeal joint with discharging sinus and another fusiform swelling of $5 \mathrm{~cm}^{*} 3 \mathrm{~cm}$ over the proximal interphalangeal joint of ring finger of right hand. There is a diffuse swelling over the dorsum of right hand as well over the third metacarpal with a discharging sinus. A small swelling was present in the right elbow and left leg also. Both the swellings were bony hard fixed to underlying bone, non-tender with no local rise of temperature. There was flexion deformity of these two fingers. Movements of fingers were restricted at the right proximal interphalangeal joint of both thumb and ring finger. On general examination patient was thin built with features of undernutrition, some pallor and multiple submandibular and submental lymphnodes were enlarged to size ranging from $1.5 \mathrm{~cm}$ to $2 \mathrm{~cm}$ all soft, non-tender, and nonmatted. On systemic examination, no significant findings were there. Other systems were within normal limits.

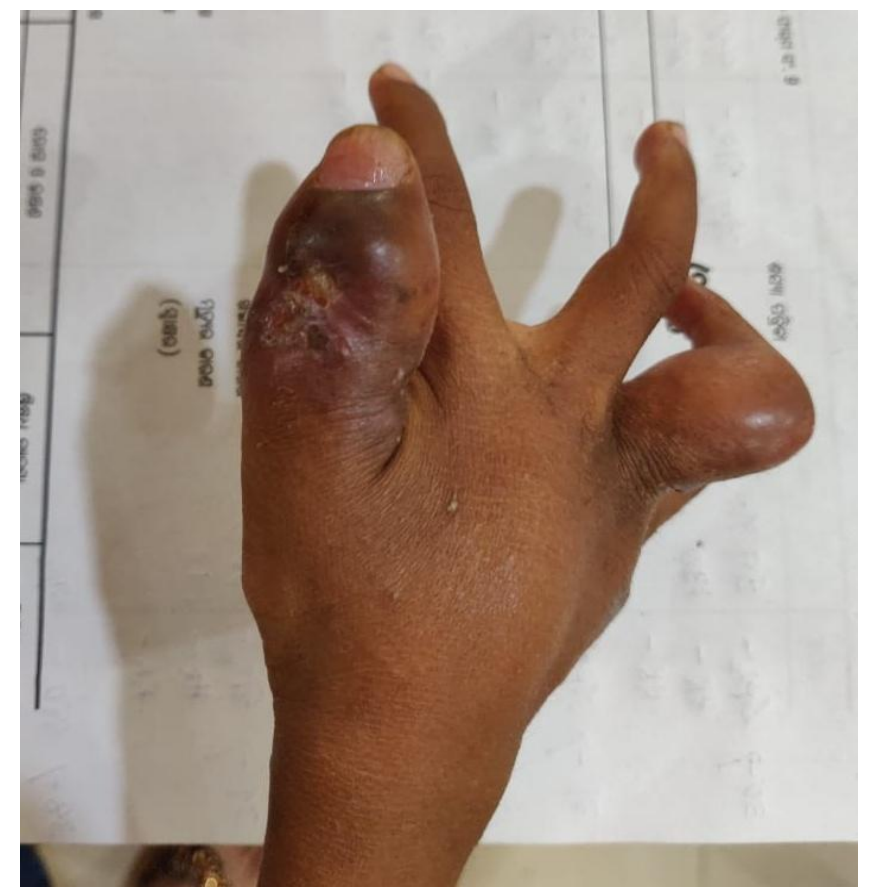

Fig 3: Showing discharging sinus over the right thumb

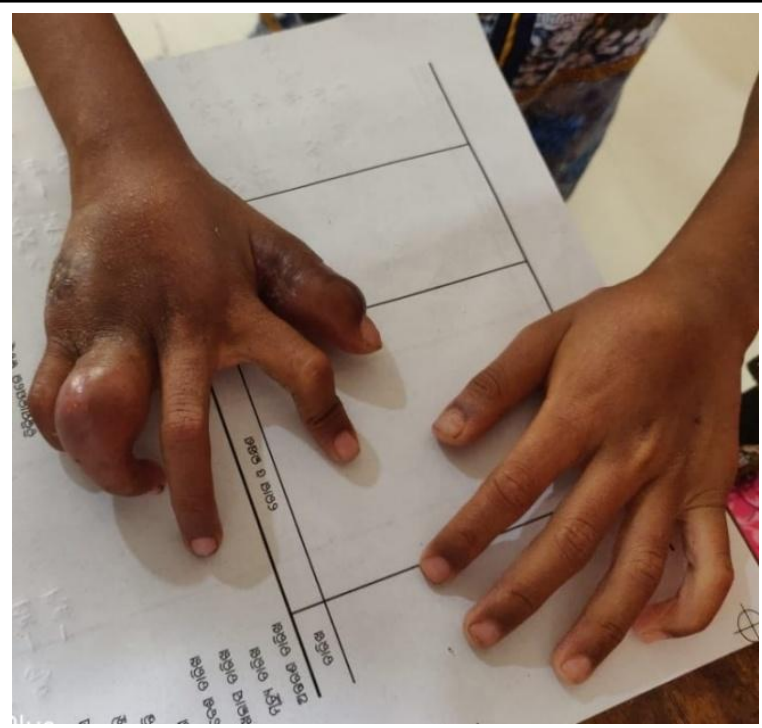

Fig 2: Showing fixed flexion deformity at metacarpophalangeal joint of right thumb and right ring finger

\section{Investigations}

Complete laboratory investigations were done in which hemogram revealed only haemoglobin of $8.5 \mathrm{~g} / \mathrm{dL}$ with microcytic hypochromic anaemia, and total leucocyte count of 8390 cells/mm3 and haematocrit of $25.6 \%$. Erythrocyte sedimentation rate was increased $(60 \mathrm{~mm} / \mathrm{hr})$. FNAC of lymphnode showed epithelioid cells with caseation necrosis suggestive of tubercular lymphadenitis. HIV was seronegative and Mantoux test was strongly positive with an in duration of $22 \mathrm{~mm}$. Sputum AFB and CBNAAT was negative and blood culture was sterile. Other blood investigations were normal.

Chest radiograph was normal. Radiograph of the right hand showed multiple lytic lesions involving proximal phalanx of thumb, cystic expansion of 3rd,and 5th metacarpals, andlytic lesions in proximal and middle phalanx of ring finger suggestive of radiological picture of tuberculous dactylitis. Antitubercular therapy was initiated under Cat 1 of RNTCP was started for 9 months with 2HRZE +7HRE. The patient is currently under follow up and is showing improvement with ATT. On follow up, there is reduction in the size of the swellings and healing of the sinuses. 


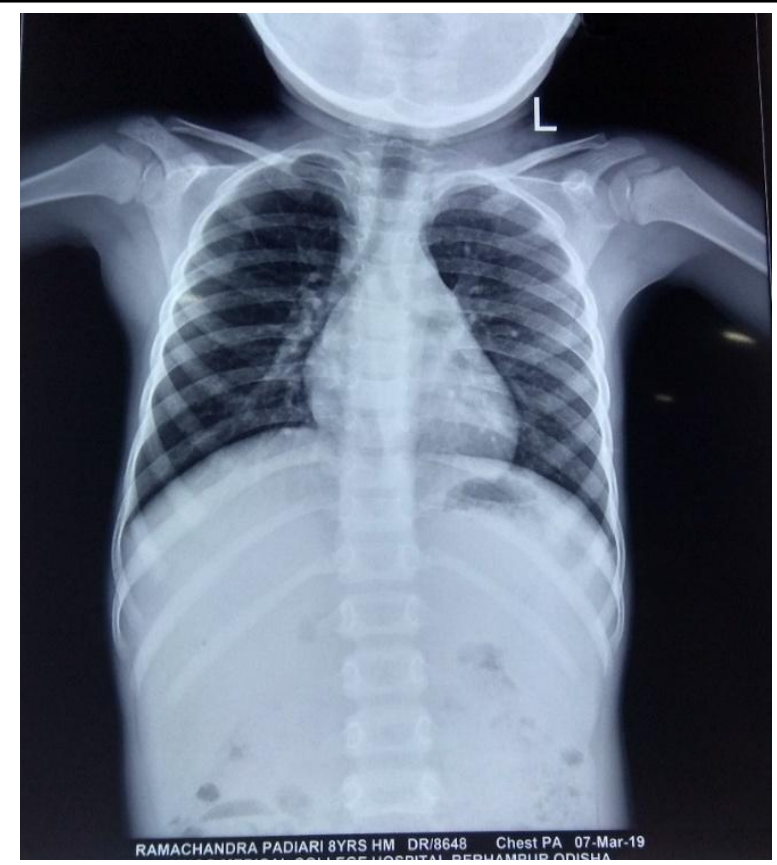

Fig 3: Showing normal chest $X$ ray without any tuberculosis focus

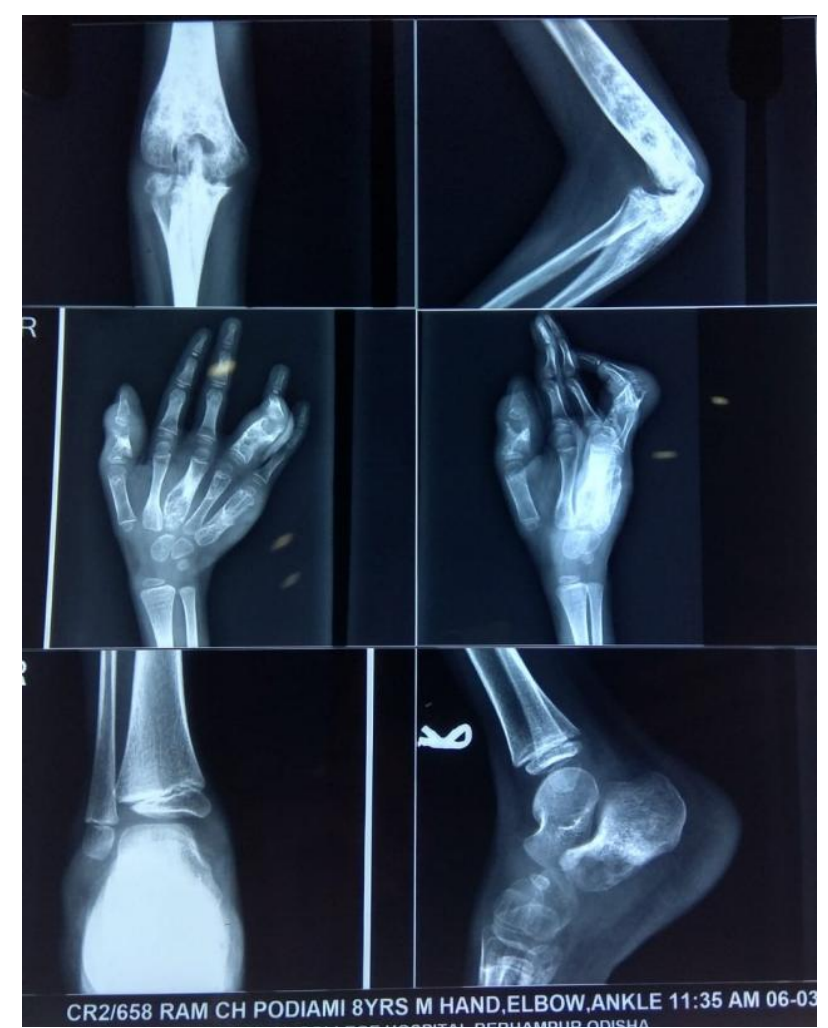

Fig 4: $\mathrm{X}$ ray showing lesions-cortical destruction, sclerosis and cystic expansion in the phalanges of right thumb; right ring finger, $3^{\text {rd }}$ and $5^{\text {th }}$ metacarpals of right hand

\section{Discussion}

Osseous tuberculosis is always the result of lympho hematogenous spread from a distant focus and it becomes symptomatic only after 1-3 years after initial infection. ${ }^{(5)}$ It occurs in $1-6 \%$ children with untreated initial pulmonary tuberculosis. $85 \%$ of the children with tuberculous dactylitis are younger than 6 years of age and incidence is reported to be $0.65 \%-6.9 \% .{ }^{(6)}$ In this age group, the hematopoietic marrow of tubular bones is favourable for hematogenous spread of tuberculosis to the marrow.

The lesion in the bone is essentially a lytic lesion which is evident radiologically and as lesion heals sclerosis takes place ${ }^{(7)}$ In short long bones of hands and feet there is intense sclerotic activity by layer of subperiosteal bone and is characteristic of tuberculous lesion. The tuberculous pus formed in the medullary canal may travel distally/laterally lifting the periosteum, may form an abscess and even burst giving rise to a tuberculous sinus. ${ }^{(8)}$

The first inoculum is of infection is lodged into in the centre of the marrow cavity and the interior of the short tubular bone is converted into a tuberculous granuloma. This results in the destruction of internal lamellae and leads to a spindle shaped expansion of the bone. The disease heals with shortening of the involved bone and occasionally with deformity and contracture of neighbouring joint.

Tuberculous dactylitis needs to be distinguished from infections like chronic pyogenic osteomyelitis and syphilitic dactylitis and neoplastic conditions with lytic lesions like enchondroma.

However, prognosis is good with antitubercular therapy and only $15 \%$ require surgery. Corrective osteotomy or excision arthroplasty is done in ankylosed joints. Significant radiological improvement is seen as early as 6 weeks. Similar cases of tuberculous dactylitis was reported by $\mathrm{S}$. Andronikou et al ${ }^{(9)}$ and by R Bandyopadhay et al. (10) Both the patients showed good response to antitubercular therapy.

High index of clinical suspicion, timely diagnosis and early institution of DOTS and routine follow up and management for complications is the key to the successful management of osseous tuberculosis. Though TB spine is the commonest 
form of osseous $\mathrm{TB}$ in older children and spina ventosa being a form of osseous TB in small children stimulated us to present this form of TB.

\section{References}

1. Tuli SM. Tuberculosis of short tubular bones Tuberculosis of the skeletal system. New Delhi: Jaypee Brothers Medical Publishers; 1991.pp. 164-5

2. Salimpour R, Salimpour P. Picture of the month. Tuberculous dactylitis. Arch Paediatr Adolesc Med1997;151:851-2.

3. Cremin BJ, Jamieson DH. Childhood Tuberculosis: modern imaging and clinical concepts.London:Springer-Verlag1995:99

4. A. Panchonia, C V Kulkarni, R Meher, S Mandwariya, Isolated tuberculous dactylitis in a 9 year old boy- a rare entity, "International Journal of Basic And Applied Medical Sciences,vol 2,52, no 20, p 55,2012

5. Kushwaha RA, Kant S, Verma SK, Sanjay Mehra S. Case report: Isolated metacarpal bone tuberculosis-A case report. Lung India.2008;25(1):17-9

6. Gyanshankar PM, Dhamgaye TM, Amol BF.Spina ventosa discharging tubercle bacilli- a case report. Indian J Tuberc.2009;56(2):00-3

7. Zoga A, Lee VW. Paediatric case of the day. Tuberculosis dactylitis and primary pulmonary tuberculosis. Am J Roentgenol1999;173:813,815-17

8. Essentials of tuberculosis in children Vimlesh Seth and S K Kabra- $3^{\text {rd }}$ edition; section D,13 Osteoarticular Tuberculosis, pg 249

9. S Andronikou, B Smith; Case report "Spina VEntosa" -tuberculous dactylitis. Archives of Diseases In Childhood 2002;86:206
10. B Andyopadhyay R, Mukherjee A, Mondal RK. Case report: "Spina Ventosa" tuberculous dactylitis in a 2 year old boyA very Rare Disease. Open Orthop J.2012:6:118-20. 